\title{
Clinical impact of routine CT esophagogram after peroral endoscopic myotomy (POEM) for esophageal motility disorders
}

다 (i) $(-)$

\author{
Authors \\ Institutions \\ 1 Department of Gastroenterology and Digestive \\ Oncology, Cochin Hospital, Assistance Publique- \\ Hôpitaux de Paris, Paris, France \\ 2 Department of Abdominal and Interventional Imaging, \\ Cochin Hospital, Assistance Publique-Hôpitaux de Paris, \\ Paris, France \\ 3 Université de Paris, Paris, France
}

Marion Chartier ${ }^{1}$, Maxime Barat ${ }^{2,3}$, Anthony Dohan ${ }^{2,3}$, Arthur Belle ${ }^{1}$, Ammar Oudjit $^{2}$, Einas Abou Ali ${ }^{1,3}$, Rachel Hallit ${ }^{1}$, Chloé Leandri ${ }^{1}$, Sophie Scialom ${ }^{1}$, Romain Coriat ${ }^{1,3}$, Stanislas Chaussade ${ }^{1,3}$, Philippe Soyer ${ }^{2,3}$, Maximilien Barret ${ }^{1,3}$

submitted 14.1 .2021

accepted after revision 10.5 .2021

\section{Bibliography}

Endosc Int Open 2021; 09: E1355-E1360

DOI 10.1055/a-1512-9638

ISSN 2364-3722

(C) 2021. The Author(s).

This is an open access article published by Thieme under the terms of the Creative Commons Attribution-NonDerivative-NonCommercial License, permitting copying and reproduction so long as the original work is given appropriate credit. Contents may not be used for commercial purposes, or adapted, remixed, transformed or built upon. (https://creativecommons.org/licenses/by-nc-nd/4.0/)

Georg Thieme Verlag KG, Rüdigerstraße 14,

70469 Stuttgart, Germany

\section{Corresponding author}

Maximilien Barret, MD, PhD, Service de Gastro-Entérologie et Oncologie Digestive, Hôpital Cochin, 27, rue du Faubourg St Jacques, 75014 Paris, France

Fax: +0033158413865

maximilien.barret@aphp.fr

\section{ABSTRACT}

Background and study aims Per oral endoscopic myotomy (POEM) of the lower esophageal sphincter has become a major treatment for esophageal motility disorders, especially achalasia. POEM can result in esophageal bleeding or perforation and pleural and mediastinal effusion. Early routine computed tomography (CT) esophagogram is frequently performed to assess these adverse events (AEs) before resuming oral food intake. We sought to evaluate the value of routine $C T$ esophagogram on postoperative day (POD) 1 after POEM.

Patients and methods This single-center retrospective study was performed in a tertiary referral center for interventional digestive endoscopy. We included consecutive patients with POEM and routine CT esophagogram on POD 1 between July 2018 and July 2019.

Results Fifty-eight patients were included in the study, $79 \%$ of whom had achalasia. Twenty patients (34\%) presented post-endoscopic AEs, including two patients with severe AEs requiring intensive care admission (one compressive pneumothorax and one mediastinitis); no deaths occurred. Of the $58 \mathrm{CT}$ esophagograms performed, only one was normal. The 57 others $(98 \%)$ showed at least one abnormal finding: pneumoperitoneum or retroperitoneal air (91\%), pneumomediastinum (78\%), pleural effusion (34\%), pneumothorax (14\%), pneumonia (7\%), pericardial effusion ( $2 \%$ ), and mediastinal collection ( $2 \%)$. CT esophagograms revealed $\mathrm{AEs}$ and modified therapeutic management in eight patients of 58 (14\%), all of whom had clinical symptoms prior to $\mathrm{CT}$.

Conclusions POD 1 CT esophagogram after POEM for esophageal motility disorders diagnosed clinically meaningful $\mathrm{AEs}$ in $14 \%$ of patients, all associated with persistent clinical symptoms. Routine use of CT esophagogram after POEM in asymptomatic patients is questionable. 


\section{Introduction}

Per oral endoscopic esophageal myotomy (POEM), is a relatively recent technique for treatment of esophageal motility disorders, and more particularly achalasia [1]. POEM yields a 2-year efficacy rate of over $90 \%$ when used as first-line treatment, higher than that of pneumatic dilatation [2], and is an alternative to surgical myotomy [3]. Indeed, POEM has a mid-term efficacy similar to laparoscopic Heller myotomy with a lower risk of severe adverse events (AEs) of about $3 \%$, compared to $7 \%$ for surgery [3].

The main AEs associated with POEM include bleeding, perforation, pleural and mediastinal fluid, and gas effusion. Their management can require emergent surgical intervention, endoscopic treatment, or remain conservative, with 24 to 72 hours of hospital monitoring, fasting, and antibiotics. Watersoluble esophagogram performed on postoperative day (POD) 1 after POEM is routinely used in many centers, followed, in case of abnormal findings, by computed tomography (CT) esophagogram [4]. The patient is kept nihil per os until the imaging is performed, and oral food intake is typically resumed after a major adverse event has been excluded. However, the superior availability of CT over conventional esophagogram has led some centers to adopt routine CT esophagogram, with conflicting results [5-7]. Currently, the optimal modality for the diagnosis of early AEs after POEM is not known.

The purpose of this study was to evaluate the clinical impact of POD 1 routine CT esophagogram after POEM.

\section{Patients and methods}

\section{Patients}

Written patient consent was obtained before the endoscopic procedure for the procedure and the use of their data for clinical research. The study was approved by the local institutional review board (CLEP NAAA-2019-08021).

This was a single-center, retrospective, observational study conducted at an expert center for interventional digestive endoscopy. We included consecutive patients who had POEM for obstructive esophageal motility disorders, followed as per our standard protocol at that time by a CT esophagogram on POD 1 between July 2018 and July 2019. We collected data on patients (age, sex, and type of esophageal motor disorder), endoscopic procedure (anterior or posterior myotomy approach, per-endoscopic AE), post-endoscopic AEs (type, severity, and management), imaging findings, and length of hospital stay. AEs were defined, as "an event that prevents completion of the procedure, and/or results in hospital admission, prolongation of existing hospital stay, another procedure needing sedation or anesthesia, or subsequent medical consultation" and graded according to the American Society of Gastrointestinal Endoscopy lexicon [8]. Following this definition, chest pain, subcutaneous emphysema, pneumoperitoneum not requiring decompression, intraprocedural bleeding, or mild chest pain were regarded as incidents and not recorded as AEs. AEs were considered early if they occurred up to 14 days post procedure, and late if they occurred any time after 14 days [8].

\section{POEM procedure}

All patients received a liquid diet the day before POEM and antibiotherapy with amoxicillin-clavulanic acid for 5 days after the procedure. Endoscopy was performed under general anesthesia with orotracheal intubation, in supine position, with a high-definition gastroscope and $\mathrm{CO}_{2}$ insufflation.

The first step consisted of an esophagogastroduodenoscopy with aspiration of possible food residue and instillation of an antiseptic chlorhexidine mouthwash on the esophageal mucosa. POEM was then performed according to the technique described by Inoue et al. [9]. Briefly, after submucosal injection of indigo carmine colored saline, a mucosal incision is performed about 10 to $12 \mathrm{~cm}$ above the esophagogastric junction (EG]), followed by creation of a submucosal tunnel by submucosal dissection up to $3 \mathrm{~cm}$ below the EJG. The myotomy was then achieved by section of the circular muscle layer, extending from $7 \mathrm{~cm}$ above the EGJ to $3 \mathrm{~cm}$ below. Finally, the mucosal incision was closed with clips and the integrity of the mucosa checked.

\section{Post endoscopic management and follow-up}

Patients were kept nihil per os with antibiotherapy (amoxicillinclavulanic acid), double-dose proton pump inhibitors, and pain medications as needed until the next day. Oral food intake was allowed only in the absence of esophageal leakage on POD 1 CT esophagogram. Patients were discharged from the hospital on day 2 . Follow-up visits were scheduled 1 and 3 months after the procedure with assessment of the procedure efficacy (using the Eckardt score) and recording of any AE.

\section{CT examination protocol}

All CT examinations were performed with a single-source 64section MDCT unit (Somatom Sensation, Siemens Healthineers). CT examinations were all performed with a triple phase protocol with and without oral contrast material. An unenhanced phase was used before and after administration of oral contrast material. Low-dose unenhanced CT images were obtained with a section thickness of $1.25 \mathrm{~mm}$, with an extended coverage of chest, to depict any preexisting hyperattenuating material [10]. Unenhanced CT was repeated using a standard radiation dose through the same coverage area with diluted non-ionic water-soluble oral contrast (Iohexol, Omnipaque; GE Healthcare; $240 \mathrm{mg}$ of iodine/mL). A third phase was obtained during the venous phase to study parenchymal and pleural enhancement. Imaging parameters were as follows: nominal section thickness, $0.625 \mathrm{~mm}$; beam pitch, 1.2; reconstruction interval, $1 \mathrm{~mm}$; tube voltage, $120-\mathrm{kV}(100 \mathrm{kV}$ for the first unenhanced phase); and tube current, 120-170 mA. Patients received 90 to $110 \mathrm{~mL}$ of contrast agent at a concentration of $350 \mathrm{mg} /$ $\mathrm{mL}$ of iodine (lomeprol, Iomeron 350; Bracco, or Xenetix 350; Guerbet), administered intravenously with an automated injector (OptiVantage, Mallinckrodt-Tyco/Healthcare) at a rate of 2.5 to $3.5 \mathrm{~mL} / \mathrm{s}$. We used an antecubital vein as the access route and an $18 \mathrm{G}$ venous catheter. 


\section{Image analysis}

CT esophagograms were initially analyzed by a panel of three board-certified radiologists who specialized in gastrointestinal radiology and had 10 to 30 years of experience. Images were specifically reviewed for the purpose of the study for presence or absence of pneumothorax, extraluminal contrast leak, mediastinal fluid collection, pleural effusion, pneumonia, pneumothorax pneumomediastinum, pneumoperitoneum, subcutaneous emphysema, and pericardial effusion in a binary fashion.

\section{Statistical analysis}

Continuous data were expressed as means \pm standard deviation (SD) or median and ranges, as appropriate. Differences in continuous variables were searched for using Mann-Whitney $U$ test and differences in categorical variables using Fisher's exact test. Statistical analyses were performed using GraphPad Prism software (La Jolla, CA, USA). $P<0.05$ was considered to indicate statistical significance.

\section{Results}

\section{Population}

Fifty-eight patients with a total of $58 \mathrm{CT}$ esophagograms were included in the study. There were 29 men and 29 women, with a median age of 57 years (range: 16-93 years). Seventy-nine percent of patients (46/58) had achalasia of the lower esophageal sphincter (type I for $48 \%$, type II for $37 \%$, and type III for $15 \%$ ) III), 7\% (4/58) a jackhammer esophagus, 7\% (4/58) a distal esophageal spasm, and $7 \%(4 / 58)$ had another indication for POEM (EG) outflow obstruction syndrome, esophageal diverticulum, or pseudoachalasia following Nissen fundoplication) $(\triangleright$ Table 1$)$.

\section{Technical outcomes and intraprocedural AEs}

POEM was technically feasible in all patients. Mucosal tears occurred in four patients $(5 / 58 ; 9 \%)$, closed by hemoclips, pneumothorax in three $(3 / 58 ; 5 \%)$ and a pneumoperitoneum requiring decompression in the endoscopy suite due to a difficult mechanical ventilation was observed in 15 (15/58; $26 \%)$ patients. Intraprocedural AEs requiring therapeutic intervention are presented in $>$ Table 1.

Subcutaneous emphysema without any therapeutic consequence occurred in 14 patients (14/58; 24\%). Intraprocedural bleeding was encountered in three patients $(3 / 58 ; 5 \%)$, all successfully managed by thermocoagulation.

\section{Remarkable clinical findings after POEM and AEs}

The median hospital stay was 2 days (range: $1-30$ days) ( $\bullet$ Table2). A remarkable clinical finding, such as dyspnea, major chest pain, or fever, occurred in 16 patients $(16 / 58 ; 28 \%$ ) on POD 1. Of these, all had abnormal CT findings and only 10 patients $(10 / 58 ; 17 \%)$ experienced an actual $\mathrm{AE}$, such as pleural effusion, pneumothorax, pneumonia, esophageal fistula, or pericardial effusion. Two AEs $(2 / 58 ; 3 \%)$, one compressive pneumothorax $(1 / 58 ; 2 \%)$, and one mediastinitis $(1 / 58 ; 2 \%)$ re-
- Table 1 Characteristics of the 58 patients and the peroral endoscopic myotomy procedure who underwent routine POD 1 CT esophagogram after POEM.

\begin{tabular}{|c|c|}
\hline Age (years) & $57(16-93)$ \\
\hline Male & $29(29 / 58 ; 50 \%)$ \\
\hline \multicolumn{2}{|l|}{ Esophageal motility disorder } \\
\hline - Achalasia & $46(46 / 58 ; 79 \%)$ \\
\hline - Type 1 achalasia & $22(22 / 58 ; 38 \%)$ \\
\hline - Type 2 achalasia & $17(17 / 58 ; 29 \%)$ \\
\hline - Type 3 achalasia & $7(7 / 58 ; 12 \%)$ \\
\hline - Other & $12(12 / 58 ; 21 \%)$ \\
\hline \multicolumn{2}{|l|}{ Orientation of the myotomy } \\
\hline - Anterior & $35(35 / 58 ; 60 \%)$ \\
\hline - Posterior & $23(23 / 58 ; 40 \%)$ \\
\hline Intraprocedural adverse event* & $20(20 / 58 ; 34 \%)$ \\
\hline - Pneumoperitoneum & $15(15 / 58 ; 26 \%)$ \\
\hline - Mucosal tear & $5(5 / 58 ; 9 \%)$ \\
\hline - Pneumothorax & $3(3 / 58 ; 5 \%)$ \\
\hline \multicolumn{2}{|c|}{$\begin{array}{l}\text { Quantitative variables are expressed as medians; numbers in brackets are } \\
\text { ranges. } \\
\text { Qualitative variables are expressed as raw numbers; numbers in parentheses } \\
\text { are proportions followed by percentages. } \\
{ }^{*} \text { Requiring therapeutic intervention. }\end{array}$} \\
\hline
\end{tabular}

- Table 2 Abnormal findings on POD 1 CT esophagogram and adverse events after POEM $(n=58)$.

- Early adverse event

$7(7 / 58 ; 12 \%)$

- Pleural effusion

$4(4 / 58 ; 7 \%)$

- Pneumonia

$2(2 / 58 ; 3 \%)$

- Esophageal fistula

$1(1 / 58 ; 2 \%)$

- Other: pericardial effusion with atrial $1(1 / 58 ; 2 \%)$ fibrillation

Late adverse event ${ }^{1}$

$4(4 / 58 ; 7 \%)$

- Abnormal CT finding on POD 1

$57(57 / 58 ; 98 \%)$

- Pneumoperitoneum

$53(53 / 58 ; 91 \%)$

- Pneumomediastinum $45(45 / 58 ; 78 \%)$

- Pneumothorax

- Pleural effusion

- Pneumonia

- Mediastinal collection

- Pericardial effusion $8(8 / 58 ; 14 \%)$ $20(20 / 58 ; 34 \%)$ $4(4 / 58 ; 7 \%)$ $1(1 / 58 ; 2 \%)$ $1(1 / 58 ; 2 \%)$

- Remarkable clinical finding after POEM

- Dyspnea/oxygen desaturation

$16(16 / 58 ; 28 \%)$

- Major chest pain ${ }^{2}$ $8(8 / 58 ; 14 \%)$

- Fever $7(7 / 58 ; 12 \%)$

$5(5 / 58 ; 9 \%)$

POD 1, first postoperative day; CT, computed tomography; POEM, per-oral endoscopic myotomy.

Data are expressed as raw numbers; numbers in parentheses are proportions followed by percentages.

${ }^{1}$ Gastroesophageal reflux

2 Requiring grade III analgesics 
quired prolonged intensive care admission and were therefore graded as severe. No deaths occurred.

Ten patients had remarkable clinical findings on POD 1 with spontaneous resolution within 24 hours, no prolonged hospital stay or specific therapeutic intervention, and therefore were not recorded as AEs. Two had major chest pain without clinical or radiological explanation (pneumoperitoneum and pneumomediastinum); three had fever, of whom two had a pleural effusion on POD 1 CT; one had dyspnea, without clinical or radiological explanation (pneumoperitoneum and pneumomediastinum); and four had subcutaneous emphysema, of whom one had a minor pneumothorax, and one a pleural effusion, while the other two patients had pneumoperitoneum and pneumomediastinum on POD 1 CT esophagogram.

\section{CT esophagogram findings}

A total of 57 CT esophagograms (57/58; $98 \%$ ) showed at least one abnormal finding, including pneumoperitoneum or retroperitoneum on $53 / 58$ (91\%), pneumomediastinum on $54 / 58$ (78\%), pleural effusion on 20/58 (35\%), pneumothorax on $8 /$ $58(14 \%)$, pneumonia on $7 \%(4 / 58)$, pericardial effusion on $2 \%$ (1/58), and mediastinal fluid collection on $1 / 58$ (2\%) CT esophagograms (> Fig. 1 and $>$ Fig.2). One CT esophagogram showed normal findings, including widened esophagus, parietal edema, clips in the lower esophagus, and the absence of oral contrast material leakage outside the esophagus.

Overall, CT esophagograms revealed post POEM AEs and resulted in altered patient management in eight of 58 patients (14\%). Of note, all eight patients had clinical symptoms, such as fever, chest pain, dyspnea or oxygen desaturation prior to the CT esophagogram. Two patients had major chest pain requiring a prolonged hospital admission and were therefore recorded as early AEs, while POD 1 CT esophagogram only showed pneumoperitoneum and subcutaneous emphysema, not explaining the patients' symptoms. We provide details on these eight patients in $>$ Table 3.

\section{Discussion}

We observed a $3 \%$ incidence of severe morbidity after POEM, at the upper limit of the $0.5 \%$ to $2.7 \%$ range reported in the literature $[3,11]$. Furthermore, while CT esophagogram appears to be a valid tool for identification of AEs after POEM, routine POD 1 CT esophagogram after POEM is unlikely to change management of asymptomatic patients and could be abandoned. Although pneumoperitoneum or even pneumothorax usually indicate severe illness requiring emergent management in other clinical settings, our data demonstrate that pneumoperitoneum and pneumothorax are expected findings after POEM, with no clinical consequences in most patients.

In six of the 16 patients with a remarkable clinical finding after POEM, the symptoms resolved spontaneously within 24 hours and they did not experience any AEs. Of the 10 with persistent (>24 hours) remarkable clinical findings, all had an $\mathrm{AE}$ and eight had a CT finding related to the AE. Therefore, to maximize the diagnostic yield, post POEM CT esophagogram should

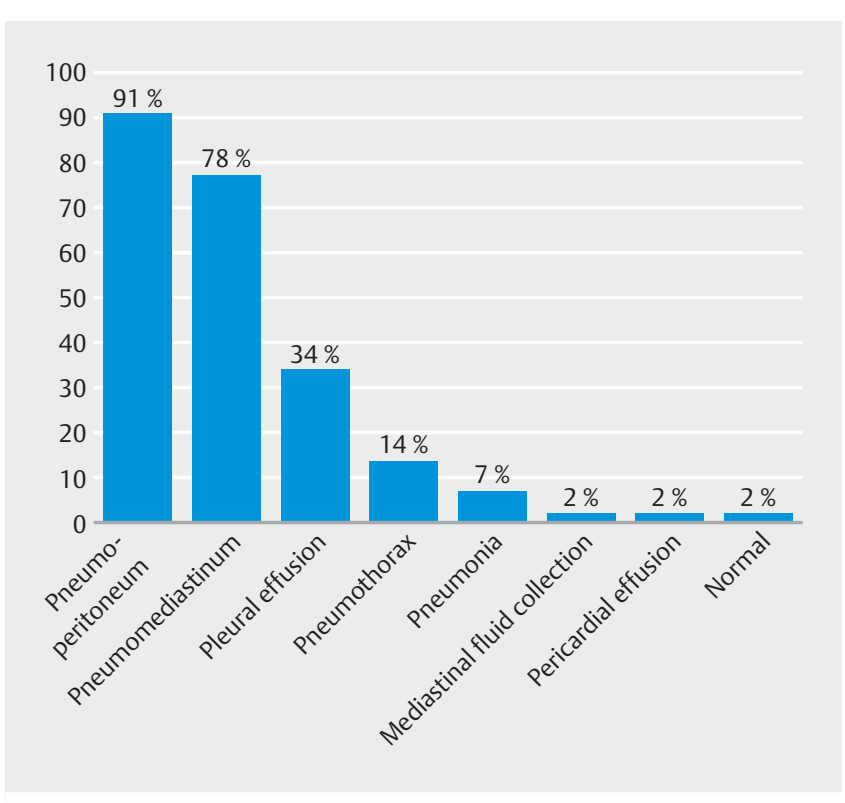

- Fig. 1 Prevalence of findings on routine POD 1 CT esophagogram after POEM in 58 patients.
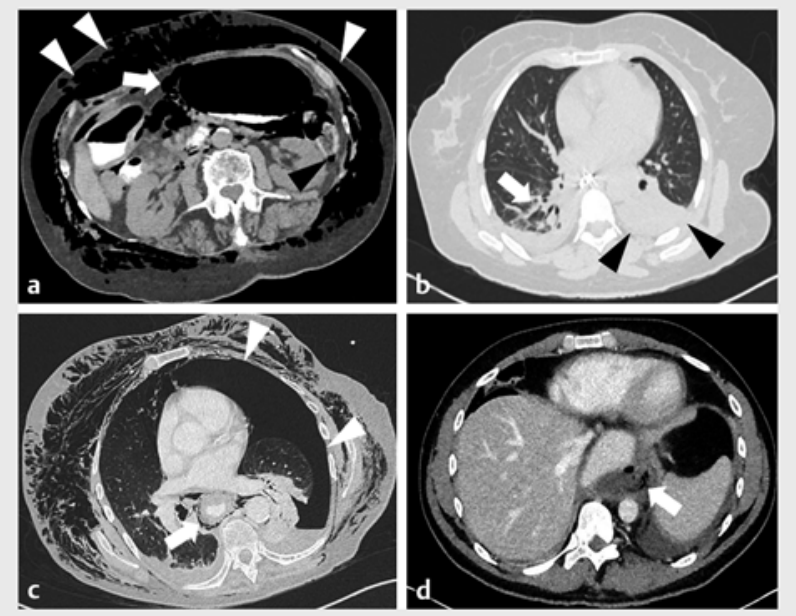

- Fig. 2 Different CT findings after POEM in four individual patients. a CT image in the axial plane reveals pneumoperitoneum (arrow), retroperitoneal air (black arrowhead), and subcutaneous emphysema (white arrowheads) after POEM for type II achalasia in a 79-year-old man without symptoms, without therapeutic consequence and uneventful follow-up. b CT image in the axial plane shows aspiration pneumonia (arrow) and pleural effusion (arrowheads) after POEM for esophagogastric junction outflow obstruction in a 54-year-old woman with chest pain, cough an oxygen desaturation, requiring antibiotic administration, oxygenotherapy, and prolonged hospital stay. C CT image in the axial plane demonstrates unilateral pneumothorax (arrowheads) and pneumomediastinum (arrow) after POEM for type III achalasia in an 86-year-old woman with dyspnea and desaturation, requiring intensive care admission and drainage. $\mathbf{d}$ CT image in the axial plane shows mediastinal collection (arrow) after redo POEM for recurrent type I achalasia in a 38-year-old man with fever and chest pain, requiring radiological and surgical management. 
- Table 3 Characteristics of the eight patients in which POD 1 CT esophagogram impacted the clinical management.

\begin{tabular}{|c|c|c|c|c|c|}
\hline $\begin{array}{l}\text { Patient } \\
\text { number }\end{array}$ & $\begin{array}{l}\text { Age, } \\
\text { sex }\end{array}$ & Clinical symptom & CT esophagogram finding & Adverse event & Management \\
\hline 1 & $\begin{array}{l}67 \text { y.o., } \\
\text { male }\end{array}$ & $\begin{array}{l}\text { Major chest pain, } \\
\text { fever }\end{array}$ & $\begin{array}{l}\text { Pneumoperitoneum, pneumomediastinum, } \\
\text { pneumothorax, pleural effusion, pneumonia }\end{array}$ & $\begin{array}{l}\text { Pleural effusion, } \\
\text { pneumonia }\end{array}$ & $\begin{array}{l}\text { Oxygen, prolonged } \\
\text { admission, antibiotics }\end{array}$ \\
\hline 2 & $\begin{array}{l}86 \text { y.o., } \\
\text { female }\end{array}$ & $\begin{array}{l}\text { Dyspnea, subcuta- } \\
\text { neous emphysema }\end{array}$ & $\begin{array}{l}\text { Pneumomediastinum, pneumothorax, pneu- } \\
\text { monia }\end{array}$ & Pneumonia & antibiotics \\
\hline 3 & $\begin{array}{l}40 \text { y.o., } \\
\text { male }\end{array}$ & $\begin{array}{l}\text { Dyspnea, subcuta- } \\
\text { neous emphysema }\end{array}$ & $\begin{array}{l}\text { Pneumoperitoneum, pneumomediastinum, } \\
\text { pneumothorax }\end{array}$ & Pneumothorax & Prolonged admission \\
\hline 4 & $\begin{array}{l}37 \text { y.o., } \\
\text { male }\end{array}$ & $\begin{array}{l}\text { Major chest pain, } \\
\text { dyspnea, fever }\end{array}$ & $\begin{array}{l}\text { Pneumoperitoneum, pneumomediastinum, } \\
\text { pleural effusion, mediastinal collection }\end{array}$ & Esophageal fistula & $\begin{array}{l}\text { ICU admission, surgical } \\
\text { drainage }\end{array}$ \\
\hline 5 & $\begin{array}{l}75 \text { y.o., } \\
\text { male }\end{array}$ & $\begin{array}{l}\text { Major chest pain, } \\
\text { subcutaneous } \\
\text { emphysema }\end{array}$ & $\begin{array}{l}\text { Pneumoperitoneum, pneumomediastinum, } \\
\text { pericardial effusion }\end{array}$ & $\begin{array}{l}\text { Pericarditis and } \\
\text { atrial fibrillation }\end{array}$ & Prolonged admission \\
\hline 6 & $\begin{array}{l}57 \text { y.o., } \\
\text { female }\end{array}$ & $\begin{array}{l}\text { Major chest pain, } \\
\text { dyspnea }\end{array}$ & Pneumoperitoneum, pneumonia & Pneumonia & $\begin{array}{l}\text { prolonged admission, } \\
\text { antibiotics }\end{array}$ \\
\hline 7 & $\begin{array}{l}85 \text { y.o, } \\
\text { female }\end{array}$ & $\begin{array}{l}\text { Dyspnea, subcuta- } \\
\text { neous emphysema }\end{array}$ & $\begin{array}{l}\text { Pneumoperitoneum, pneumomediastinum, } \\
\text { pneumothorax, pleural effusion }\end{array}$ & Pneumothorax & $\begin{array}{l}\text { ICU admission, } \\
\text { drainage }\end{array}$ \\
\hline 8 & $\begin{array}{l}78 \text { y.o., } \\
\text { female }\end{array}$ & Dyspnea & $\begin{array}{l}\text { Pneumoperitoneum, pneumomediastinum, } \\
\text { pneumothorax }\end{array}$ & Pneumothorax & $\begin{array}{l}\text { Oxygen, prolonged } \\
\text { admission }\end{array}$ \\
\hline
\end{tabular}

be performed in patients with remarkable clinical findings that perist for $>24$ hours.

Three studies have specifically studied the role of routine CT after POEM [5-7]. Pannu et al. prospectively included 84 consecutive patients and observed pneumomediastinum in $85.7 \%$ of patients, pneumoperitoneum in $66.7 \%$, pleural effusion in $46.4 \%$, and pneumothorax in $19 \%$ [5]. In their study, five patients required an intervention based on CT findings, among whom four patients had pneumonia and one had an esophageal leak [5]. Noticeably, CT revealed an esophageal leak before any clinical manifestation [5]. In a study involving 108 patients who underwent routine POD 1 CT esophagogram after POEM, Yang et al. reported pneumoperitoneum or pneumomediastinum in $53 \%$ of patients, and pleural effusion in $69.4 \%$. They did not show any association between pneumoperitoneum or pneumomediastinum and the occurrence of an $\mathrm{AE}$ and concluded that these imaging findings are normal postoperative changes [6]. Indeed, once the myotomy is completed, the $\mathrm{CO}_{2}$ insufflated via the endoscope may diffuse into the abdomen, mediastinum, pleura, pericardium, and subcutaneous space. However, $\mathrm{CO}_{2}$ needs a few days to resorb; hence, its presence at POD 1 does not indicate actual perforation of the esophagus. Cai el al. analyzed 300 CT esophagograms after POEM, and observed pneumomediastinum in $48 \%$ of patients, a pneumoperitoneum in $37 \%$, a subcutaneous emphysema in $28 \%$, a pneumothorax in $17 \%$, pleural effusion in $66 \%$, pneumonia in $52 \%$, and focal atelectasis in $21 \%$ [7]. Only $6.3 \%$ of CT findings had therapeutic consequences, and only one esophageal hematoma was observed on CT before any clinical manifestation [7]. The high rate of $A E s$ diagnosed in this study can be explained by the use of air insufflation in half of the patients and by a selection bias, since CT was performed in only $70 \%$ of patients with POEM, suggesting inclusion bias and possibly overestimation of the actual prevalence of AEs. Our study is the first to correlate, in consecutive patients, clinical data and routine POD 1 post POEM imaging findings to help guide clinical management of patients with clinical symptoms after POEM.

Conventional water-soluble esophagogram on POD 1 after POEM was recommended by Inoue et al [12]. Its diagnostic contribution has also been demonstrated by several groups [4, 1315]. In the aggregate, routine conventional water-soluble esophagogram revealed pneumoperitoneum in $72 \%$ to $84.9 \%$ of patients, pleural effusion in $11.3 \%$, and esophageal leakage in $1.8 \%$ to $3.8 \%[4,13,14,16]$. However, conventional water-soluble esophagogram is less rapidly available than CT esophagogram in our institution, which is why we usually perform CT esophagogram as a routine examination instead of conventional water-soluble esophagogram. Furthermore, any major abnormal finding observed on esophagogram usually require further CT examination to guide therapeutic management.

Routine POD 1 endoscopy has also been proposed, with the idea of closing inadvertent mucosotomies or placing additional clips in case of migrated clips at the mucosal entry site. This approach has been abandoned due to its invasiveness and low contribution to patient management $[4,12,14]$.

The main limitation of our work is its retrospective nature, although all patients were consecutive and submitted to a unique post POEM care protocol. In addition, the relatively small sample size, the participation of up to five endoscopists performing POEM resulting in a limited experience of each opera- 
tor, and the limited experience of the radiologists with postPOEM CT findings should be kept in mind when interpreting the study findings.

\section{Conclusions}

In conclusion, our study shows that routine POD 1 CT esophagogram after POEM reveals AEs altering patient management in $14 \%$ of patients, raising major concerns regarding the utility of routine CT esophagogram both from a clinical and cost-efficiency point of view. Most importantly, POD 1 CT esophagogram helped in the clinical management only in patients with clinical symptoms that persisted for $>24$ hours. Our results, in line with those of other researchers [7], suggest restricting the use of CT esophagogram after POEM to patients with major chest pain, fever, dyspnea, cough or oxygen desaturation that persists 24 hours after the procedure.

\section{Acknowledgements}

The authors thank Dr. Olivier Cerles from the University of Paris for his assistance in editing and proofreading of their paper.

\section{Competing interests}

The authors declare that they have no conflict of interest.

\section{References}

[1] Kahrilas PJ, Katzka D, Richter JE. Clinical Practice Update: The use of per-oral endoscopic myotomy in achalasia: expert review and best practice advice from the AGA Institute. Gastroenterology 2017; 153: 1205-1211

[2] Ponds FA, Fockens P, Lei A et al. Effect of peroral endoscopic myotomy vs pneumatic dilation on symptom severity and treatment outcomes among treatment-naive patients with achalasia: a randomized clinical trial. JAMA 2019; 322: 134-144
[3] Werner YB, Hakanson B, Martinek J et al. Endoscopic or surgical myotomy in patients with idiopathic achalasia. N Engl J Med 2019; 381: 2219-2229

[4] Barret M, Dohan A, Oudjit A et al. Meglumine diatrizoate esophagogram after peroral endoscopic myotomy (POEM): identification of imaging findings associated with clinical complications and longer hospital stay. Eur Radiol 2020; 30: 4175-4181

[5] Pannu D, Yang D, Abbitt PL et al. Prospective evaluation of CT esophagram findings after peroral endoscopic myotomy. Gastrointest Endosc 2016; 84: 408-415

[6] Yang S, Zeng M-S, Zhang Z-Y et al. Pneumomediastinum and pneumoperitoneum on computed tomography after peroral endoscopic myotomy (POEM): postoperative changes or complications? Acta Radiol 2015; 56: 1216-1221

[7] Cai M-Y, Zhou P-H, Yao L-Q et al. Thoracic CT after peroral endoscopic myotomy for the treatment of achalasia. Gastrointest Endosc 2014; 80: 1046-1055

[8] Cotton PB, Eisen GM, Aabakken L et al. A lexicon for endoscopic adverse events: report of an ASGE workshop. Gastrointest Endosc 2010; 71: 446-454

[9] Inoue H, Minami H, Kobayashi Y et al. Peroral endoscopic myotomy (POEM) for esophageal achalasia. Endoscopy 2010; 42: 265-271

[10] Greffier J, Pereira F, Hamard A et al. Effect of tin filter-based spectral shaping $\mathrm{CT}$ on image quality and radiation dose for routine use on ultralow-dose CT protocols: A phantom study. Diagn Interv Imaging 2020; 101: 373-381

[11] Haito-Chavez Y, Inoue H, Beard KW et al. Comprehensive Analysis of adverse events associated with per oral endoscopic myotomy in 1826 patients: an international multicenter study. Am J Gastroenterol 2017; 112: 1267-1276

[12] Inoue $\mathrm{H}$, Sato $\mathrm{H}$, Ikeda $\mathrm{H}$ et al. Per-oral endoscopic myotomy: a series of 500 patients. J Am Coll Surg 2015; 221: 256-264

[13] Levy JL, Levine MS, Rubesin SE et al. Findings of esophagography for 25 patients after peroral endoscopic myotomy for achalasia. AJR Am J Roentgenol 2016; 207: 1185-1193

[14] Nast JF, Berliner C, Rösch T et al. Endoscopy versus radiology in postprocedural monitoring after peroral endoscopic myotomy (POEM). Surg Endosc 2018; 32: 3956-3963

[15] Reddy CA, Tavakkoli A, Abdul-Hussein M et al. Clinical impact of routine esophagram after peroral endoscopic myotomy. Gastrointest Endosc 2021; 93: 102-106

[16] Harmath C, Horowitz J, Berggruen S et al. Fluoroscopic findings postperoral esophageal myotomy. Abdom Imaging 2015; 40: 237-245 